Mens

revue d'histoire intellectuelle de l'Amérique française

\title{
Harry Bernard (1898-1979) : érudit et homme de lettres
}

\section{Guy Gaudreau et Micheline Tremblay}

Volume 2, numéro 1, automne 2001

URI : https://id.erudit.org/iderudit/1024457ar

DOI : https://doi.org/10.7202/1024457ar

Aller au sommaire du numéro

Éditeur(s)

Centre de recherche en civilisation canadienne-française

ISSN

1492-8647 (imprimé)

1927-9299 (numérique)

Découvrir la revue

Citer cet article

Gaudreau, G. \& Tremblay, M. (2001). Harry Bernard (1898-1979) : érudit et homme de lettres. Mens, 2(1), 35-65. https://doi.org/10.7202/1024457ar

\section{Résumé de l'article}

Cet article trace la biographie du journaliste Harry Bernard (1898-1979). À partir du dépouillement de sa volumineuse correspondance, déposée à la Bibliothèque nationale du Québec, et des nombreux articles qu'il publia au cours de sa carrière au Droit et au Courrier de Saint-Hyacinthe, le portrait esquissé dévoile un itinéraire professionnel complexe où se juxtaposent des écrits de toutes sortes. Sa biographie constitue une contribution originale à l'étude de l'évolution des idées au Canada français entre 1920 et 1960. Aujourd'hui totalement oublié - sauf de quelques historiens littéraires -, Harry Bernard reste toutefois un important érudit et homme de lettres de son époque. 


\section{HARRY BERNARD (1898-1979) : ÉRUDIT ET HOMME DE LETTRES}

GUY GAUDREAU

Département d'histoire

MICHELINE TREMBLAY

Département d'études françaises

Université Laurentienne

\section{RÉSUMÉ}

Cet article trace la biographie du journaliste Harry Bernard (1898-1979). À partir du dépouillement de sa volumineuse correspondance, déposée à la Bibliothèque nationale du Québec, et des nombreux articles qu'il publia au cours de sa carrière au Droit et au Courrier de Saint-Hyacinthe, le portrait esquissé dévoile un itinéraire professionnel complexe où se juxtaposent des écrits de toutes sortes. Sa biographie constitue une contribution originale à l'étude de l'évolution des idées au Canada français entre 1920 et 1960. Aujourd'hui totalement oublié - sauf de quelques historiens littéraires -, Harry Bernard reste toutefois un important érudit et homme de lettres de son époque.

\section{ABSTRACT}

This article outlines the life of journalist Harry Bernard (1898-1979). Resting on research conducted at Quebec's National Library, where Bernard's voluminous correspondence can be consulted, and the considerable number of articles he published during his career as a journalist at Ottawa's Le Droit and at the Courrier de Saint-Hyacinthe, the present biography traces the portrait of a man whose complex professional itinerary was characterized by the juxtaposition of several literary genres. Bernard's biography offers an original contribution to the study of the evolution of the French Canadian mind between 1920 and 1960. Though contemporary scholars - 
save for a few literary historians - have largely forgotten him, Harry Bernard was a key figure in the intellectual life of his era.

Harry Bernard. Son nom a été pour ainsi dire effacé de la mémoire collective. Pourtant, il est demeuré actif pendant plus de cinquante ans en menant une longue carrière en tant que rédacteur en chef au Courrier de Saint-Hyacinthe et ce, parallèlement à la publication de nombreux ouvrages romanesques et savants. En fait, cet homme de lettres fut à la fois journaliste, éditorialiste, romancier, critique littéraire, vulgarisateur scientifique et poète.

Les biographies publiées jusqu'à maintenant ne lui rendent pas justice; elles se résument à quelques paragraphes et les dictionnaires spécialisés ne contiennent guère plus de détails ${ }^{1}$. Quant aux manuels d'histoire littéraire, ils demeurent tout aussi avares de commentaires, lorsqu'ils lui consacrent une notice biographique ${ }^{2}$ ! Selon Gaston Désaulniers, auteur d'un mémoire de maitrise sur son oeuvre romanesque, Bernard «a connu, à la parution de ses livres, des heures de gloire qui ne pouvaient laisser présager l'oubli presque total dans lequel a sombré son oeuvre romanesque ${ }^{3} . »$ «Qui parle aujourd'hui de Bernard?», écrivait récemment Robert Lahaise 4 . Comment expliquer que ce lauréat de trois prix David qui a signé pendant trente-cinq ans des critiques littéraires, publié divers travaux sur les mammifêres et les poissons, qui s'est impliqué dans la vie sociale de son temps que ce soit en tant que pionnier de L'Action nationale, en agissant avec force pour appuyer et développer le livre canadien ou en menant de virulents combats pour défendre ses positions politiques, soit aujourd'hui à ce point méconnu?

Pourquoi cet oubli? Pourquoi a-t-il été écarté par les historiens des idées et ceux de la littérature? Sans doute parce qu'il fait partie de ces auteurs nationalistes, de ces journalistes catholiques qui ont tant dominé le Canada français d'avant la Révolution tranquille et que l'on s'est empressé de rejeter au cours des années 1960. En l'écartant, c'est tout le courant dominant des lettrés «en service national» que l'on a occulté, pour ne privilégier souvent que les avantgardistes et les anticléricaux ${ }^{5}$. Pourtant, s'il est un homme de lettres 
dont on devrait connaître la carrière afin de comprendre l'évolution des idées au Canada français, c'est bien lui. En effet, à l'image de plusieurs d'entre eux, il est journaliste; ses romans sont des oeuvres moyennes, pour reprendre l'expression de Daniel Chartier ; comme tant d'autres, il est disciple de Groulx et ses positions politiques unionistes rejoignent une bonne partie de la population qui vote pour Duplessis. D'ailleurs, l'appui d'un homme de lettres comme Bernard au régime Duplessis jette un nouvel éclairage sur l'emprise que pouvait exercer ce premier ministre populiste.

Une notice biographique consacrée à Bernard constitue une contribution originale à l'étude des idées au Canada français d'avant les années 1960. L'homme apparaît sous de multiples visages qui interdisent les raccourcis et les simplifications. Homme d'action, il ne se contentait pas d'écrire en retrait de la réalité. Et ce n'est pas parce que ses écrits paraissent aujourd'hui démodés qu'ils s'inscrivent dans un parcours inintéressant.

Par la même occasion, nous souhaitons faire connaître un projet de recherche plus vaste sur Harry Bernard. Parmi les volets déjà prévus, il y aura sa conception régionaliste de la littérature, la réception critique de ses oeuvres ${ }^{7}$, l'étude fouillée de son rapport au cinéma et de son rapport à la politique, l'examen de sa riche correspondance avec Alfred DesRochers, l'analyse des critiques littéraires qu'il rédigea au cours de la première partie de sa vie, puis celle des critiques hebdomadaires qu'il signa entre 1941 et 1971 sous le pseudonyme de «L'Illettré» et finalement, ses efforts de diffusion des sciences naturelles.

Si l'approche biographique s'avère nécessaire, c'est également parce que les auteurs précédents n'avaient pas eu accès aux archives personnelles de Harry Bernard, déposées à la Bibliothèque nationale du Québec en 1980 , pour lequel un répertoire vient d'ailleurs de paraître ${ }^{8}$. Ce fonds totalise plus de 9,3 mètres linéaires de documents dont plus d'un mètre pour la seule correspondance, soit environ sept mille pages. En plus de sa correspondance personnelle et professionnelle - que nous avons 
été les premiers à dépouiller ${ }^{9}$-, nous avons systématiquement examiné ses nombreux articles, comptes rendus, éditoriaux publiés quotidiennement dans Le Droit et de façon hebdomadaire dans $L e$ Courrier de Saint-Hyacinthe de même qu'épisodiquement dans d'autres revues de son époque.

\section{De l'enfant au jeune adulte}

Harry Bernard est né à Londres en Angleterre le 9 mai 1898 de parents canadiens-français. Son prénom lui causera quelques soucis et il devra défendre à de nombreuses occasions ses origines canadiennes-françaises. Ainsi à la première page du Courrier, il affirme l'origine française de son prénom ${ }^{10}$. Encore en 1964, il fait la leçon à un journaliste de Québec, Rémi d'Anjou : «un homme de votre culture ne saurait ignorer qu'un saint évêque de France, bel et bien canonisé, qui vécut en des temps très anciens, s'appelait Harry".$»$

Son père, Horace Bernard, commerçant et importateur, profitait de son métier pour voyager, surtout en Europe (Angleterre, France et Suisse) et aux États-Unis (Boston). C'est d'ailleurs dans ce contexte que Harry Bernard naît à Londres alors que son père y réside, mandaté par son employeur new-yorkais. Il devait y surveiller les arrivages de mil récolté au Québec. «Je fus élevé au foin, comme les chevaux. Dans ce sens que mon père et sa famille comptaient pour vivre sur le commerce de ce fourrage ${ }^{12}$.» Peu après sa naissance, ils déménagent à Soissons, puis à Paris. Et c'est là qu'il fit ses débuts à l'école, au Collège Rollin, non loin de Montmartre. La famille y demeure jusqu'en 1904 alors que le jeune Harry est âgé de six ans. Après un court séjour à Montréal, les Bernard s'installent en Nouvelle-Angleterre et la fréquentation de l'école de St-Albans au Vermont permet à Harry de se familiariser avec l'anglais. En 1906, c'est le retour définitif au Québec d'abord à Upton, dans les Cantons de l'Est, puis à Saint-Hyacinthe à compter de 1911.

Sa famille, sans être riche, est assurément petite bourgeoise : «Ma mère fut à la belle époque le type à peu près parfait de la 
bourgeoisie moyenne, ni trop argentée ni démunie ${ }^{13} . »$ Quant à son père, l'automobile met fin à son travail d'expert en fourrage en réduisant le nombre d'animaux de trait; il doit se recycler dans l'importation et la production de bijoux de luxe. C'est à Boston qu'il ouvre son nouveau commerce en $1918^{14}$. Mais la crise des années 1930 l'oblige à fermer boutique et à congédier son personnel, y compris Émile, son fils cadet. «J'ai maintenant abandonné l'idée de devenir riche...», confie-t-il à Harry en décembre $1930^{15}$. C'est pourquoi il revient à Saint-Hyacinthe et accepte un travail à L'Union Saint-Joseph en tant que comptable.

De 1911 à 1919, Harry Bernard est inscrit au Séminaire de Saint-Hyacinthe où il terminera avec succès son cours classique. «En marge de thèmes et versions, dit-il, je rime des centaines de vers. Fatras dont il ne reste rien. Excellent exercice, le vers m'oblige cependant au mot juste, à la pensée ramassée. Le jour où Hugo m'apparaît, je brûle mes poèmes. Le jour où je lis Baudelaire, je déchire les vers inspirés par Hugo ${ }^{16}$.»

D'après des notes manuscrites de l'abbé Pierre-Athanase Saint-Pierre, «s'il ne fut pas à la tête de sa classe, il en fut l'un des premiers et en juin 1912, il remporta plusieurs accessits. [...] En rhétorique, il obtint le premier prix de discours français ${ }^{17}$.» Déjà, tout en étudiant, il collabore au Courrier de Saint-Hyacinthe en y publiant des poèmes et en s'occupant de la page «Fémina». Il n'a donc que dix-huit ans quand il fait ses débuts en tant que journaliste sous le pseudonyme de Roger Raymond. Déjà, il réfléchit sur la littérature en tant que telle et affirme avec conviction la nécessité d'en développer une qui soit authentiquement canadienne-française.

Le journalisme ne le quittera plus. Au cours de l'été 1918, Bernard s'engage dans le corps étudiant de l'armée américaine, à Lowell (Massachusetts), tandis que son père vient d'emménager à Boston pour y ouvrir son nouveau commerce.

\section{Journaliste au Droit et rédacteur au Courrier}

Après son baccalauréat, à l'automne 1919, Bernard s'installe brièvement à Montréal. Mais dès décembre, il décroche un emploi 
régulier à Ottawa. Le journal Le Droit lui offre alors un salaire de quinze dollars par semaine, avec une augmentation prévue de cinq dollars après six mois. Le hasard faisant bien les choses, il finit par loger dans le même immeuble que Léo-Paul Desrosiers et Claude Melançon avec qui il se liera d'amitié.

Au moment où il débute au Droit, en décembre 1919, Donat Kavanagh dirige le service d'information et forme les jeunes journalistes au nombre desquels se trouvent Harry Bernard et Edgar Boutet. Bien qu'il soit rattaché au service de l'information, il y publiera des contes et des poèmes. Pendant quatre ans, il circule d'une tâche à l'autre : nouvelliste (rubriques «Au jour le jour» et «Fléchette»), correspondant parlementaire aux Communes ${ }^{18}$, mais surtout adjoint au nouveau rédacteur en chef Charles Gautier, dès l'été 1920 , ce qui l'amène à signer quelques éditoriaux et à assumer le rôle de rédacteur en chef pendant les congés de Gautier. Il y agit même à titre de traducteur. Ses différentes fonctions l'amènent à traiter de sujets aussi variés que le budget fédéral, la situation irlandaise, le théâtre, la franc-maçonnerie, la dépopulation rurale, l'émigration aux États-Unis, le divorce, la presse catholique et les capitalistes ainsi que le vote des femmes. Qu'il aborde des sujets politiques, sociaux ou culturels, ses textes, bien documentés, présentent toujours un point de vue personnel, souvent ambigu, parce qu'il témoigne à la fois de la pensée de l'élite conservatrice et de son désir de transformer ou du moins de s'adapter à l'évolution de la société. Ainsi, dans un article portant sur le vote des femmes, il affirme d'abord qu' «[e]n principe, le suffrage féminin est mauvais et condamnable. Il l'est d'ailleurs comme le suffrage universel lui-même, dont il est la conséquence logique ${ }^{19} \gg$. Au fil de l'article, cependant, il nuance son assertion et conclut par ces mots : «Les femmes peuvent et doivent voter. [...] Nous ne dirons pas, loin de là, que l'intervention des femmes dans la politique est un bien, mais, puisque nous l'avons, il faut savoir en tirer la plus grande somme de bien possible ${ }^{20} . »$

En plus de ce travail au Droit, il publie des poèmes dans $L a$ Revue moderne, La Revue nationale, Les Annales d'Ottawa, 
L'Almanach littéraire de l'Est et signe, à l'occasion, des articles dans d'autres journaux comme Le Courrier de Saint-Hyacinthe.

Il rencontre à Ottawa Louella Tobin, fille de feu John Tobin. Elle devient rapidement amoureuse de lui. C'est d'ailleurs elle, semble-t-il, qui lui fait la grande demande ${ }^{21}$. Et c'est ainsi que, le 16 avril 1923, Le Droit annonce la bénédiction de son mariage. Son salaire passe alors à vingt-deux dollars par semaine, ce qui était peu, compte tenu de ses nouvelles responsabilités. Le couple aura deux filles, une née en 1924, Louella, et une autre en 1933, du nom de Marcelle. Le couple se sépare vers la fin des années 1930. Le mariage fut par moments houleux, mais Bernard a laissé peu de témoignages de sa vie amoureuse.

Outre un salaire insuffisant, d'autres facteurs vont le pousser à quitter $L e$ Droit $^{22}$. Il y a cette possibilité trop lointaine d'obtenir le poste de rédacteur en chef, étant donné que Gautier n'est que de cinq ans son aîné. Le retour de Fulgence Charpentier qui devient aussi adjoint au rédacteur ne simplifie pas la situation. Comme $L e$ Courrier de Saint-Hyacinthe vient de fusionner à La Tribune, le poste de rédacteur en chef s'ouvre et il le décroche, ce qui lui permet de revenir dans la ville de ses ancêtres et d'occuper un poste lui donnant non seulement plus de responsabilités mais aussi plus de latitude. Il débute le 4 juin 1923 et y restera quarante-sept ans, soit jusqu'au $1^{\text {er }}$ juin 1970. De vingt-cinq à soixante-douze ans, sa destinée est liée à celle de ce journal qui lui assure son gagne-pain. Il contribue à en rehausser la qualité.

\section{Une période d'intense activité littéraire : 1923-1933}

Les dix prochaines années voient l'explosion littéraire de Bernard. Ses romans, qu'il qualifie lui-même de régionalistes et non de romans du terroir ${ }^{23}$ sont ancrés dans le milieu canadien, que celuici soit urbain, comme Saint-Hyacinthe dans L'Homme tombé ou Ottawa dans La Maison vide ou bien rural comme dans La ferme des pins et La terre vivante. L'essentiel, pour lui, c'est de s'inspirer 
des paysages, du climat, des moeurs canadiens et cela inclut même le langage qui doit correspondre à celui utilisé au Canada français au lieu d'imiter le bon parler français de France. Ainsi, notre littérature se démarquera de la littérature française et extériorisera véritablement l'âme du peuple.

Il publiera six romans, un recueil de nouvelles et un autre de critiques $^{24}$. Et si ses romans ont pris le chemin des oubliettes, il n'en demeure pas moins qu'il rafla plusieurs distinctions : trois fois, il remporte le prestigieux Prix David (soit pour L'Homme tombé; pour La terre vivante et pour Juana, mon aimée ${ }^{25}$ ) et six fois celui du Concours d'Action intellectuelle (organisé par l'Action catholique de la jeunesse canadienne) avant l'âge de trente-cinq ans, âge limite fixé pour son obtention. Tout comme ses éditoriaux au Droit, ses romans témoignent de cette ambivalence entre une idéologie conservatrice aux valeurs morales strictes et austères et un net besoin de s'en démarquer. Dans L'Homme tombé, par exemple, le personnage principal croule sous l'influence d'une femme mondaine dont les après-midi se passent au cinéma et pour qui les vedettes américaines représentent un mode de vie des plus enviables. Si cet aspect de l'histoire cadre admirablement bien avec les valeurs traditionnelles, cela ne doit pas nous dissimuler que Harry Bernard fut l'un des premiers romanciers à prendre conscience de l'importante influence du cinéma sur le comportement humain et, conséquemment, sur l'évolution de la mentalitéé

Tout en explorant l'écriture romanesque, Bernard publie également dans d'autres journaux et revues ${ }^{27}$. Au Courrier de SaintHyacinthe, non seulement signe-t-il l'éditorial, mais il s'occupe aussi de la chronique «En marge des événements», calquée sur «Au jour le jour» du Droit et dans laquelle il aborde divers sujets sociaux et notamment les questions littéraires.

En 1928, jugeant sans doute la littérature trop à l'étroit sous cette rubrique dont elle partage la vedette avec tous les autres sujets de l'actualité, il en implante une nouvelle : «Le Courrier littéraire». Son but? Présenter des livres aux lecteurs 
afin de les inciter à lire davantage. Bien qu'il y fasse la recension de toutes sortes de livres (documents touristiques, relations de voyage, etc.) il y privilégie la littérature canadienne-française. «Le Courrier littéraire» se transformera en «Livres nouveaux» le 30 mai 1930. Toutefois, les articles informatifs publiés sous cette nouvelle appellation n'étant généralement pas signés, on ne peut les lui attribuer. Et ce n'est plus qu'occasionnellement, en éditorial, qu'il se penche sur certains ouvrages généraux.

Afin de donner un aperçu de ses critiques littéraires, présentons les textes qu'il publie en 1929. Dans Le Courrier de Saint-Hyacinthe, on compte vingt-cinq articles, la plupart sous la rubrique «Le courrier littéraire». L'important, pour lui, c'est de faire lire les gens afin de rehausser la culture du peuple canadien-français. Cela se reflète dans l'ouverture de ses textes à des livres non littéraires tels Les papillons diurnes de la province de Québec de V.-A. Huard, Les Généalogies des familles du Richelieu de G.-A. De Jordy ou Nos intérieurs de demain de J.-M. Gauvreau, livres qui traitent respectivement d'entomologie, de généalogie et du meuble. Voulant aussi susciter la réflexion, il signale et commente la parution d'ouvrages se préoccupant d'éducation, de philosophie, de langue, d'histoire. Il reste toutefois que c'est aux oeuvres littéraires qu'il consacre la majorité de ses textes. On note un changement important par rapport à ses débuts, dans les années 1916-1919: à quelques exceptions près, il se restreint aux ouvrages poétiques ou romanesques canadiens-français, indiquant ainsi sa volonté de développer la littérature canadienne et de la faire lire.

Cette même année, il publiera son seul ouvrage critique ${ }^{28}$ qui se compose d'articles de fond dont certains ont déjà été publiés tels ceux sur la jeune poésie, l'idée baudelairienne au Canada, le régionalisme, la langue et la culture etc. Un certain nombre d'écrivains canadiens y verront également une critique de leur oeuvre : Blanche Lamontagne, Louis Dantin, Jules Fournier, Robert deRoquebrune, Jean Chauvin, Émile Coderre de même que deux écrivains français, 
soit Émile Baumann et Charles Silvestre. Cette esquisse rapide de ses activités critiques durant l'année 1929 marque bien l'étendue de ses connaissances et ses préoccupations non seulement pour la littérature canadienne-française mais pour tout ce qui s'écrit.

Mais il trouve hasardeux de faire de la critique. En réponse à Jean-Charles Harvey qui lui dit avoir songé à abandonner la critique des livres canadiens car il «comptait tellement d'amis parmi les auteurs [qu'il se voyait] dans l'impossibilité d'être vrai et franc» ${ }^{29}$, Bernard écrit qu'il comprend son hésitation:

Je vous avoue que j'ai souvent ressenti la même impression de malaise, et je me demande souvent, comme vous, ce qu'il est opportun de faire: ou faire de la littéraire honnête, ou perdre ses amis? Je ne suis pas prêt à répondre. Evidemment, si l'on ne se place qu'au point de vue de l'art, la première solution s'impose. Mais ce n'est pas là toute la question. Il faudrait trouver un moyen terme, qui respectât ceci comme cela ${ }^{30}$.

Est-ce pour cela qu'il abonnera la critique littéraire pendant presque une dizaine d'années?

Son mentor, Lionel Groulx, relit ses textes et les commente abondamment, l'obligeant parfois à réécrire de larges parties de ses romans ${ }^{31}$. Dans ses Mémoires, Groulx précise que leur correspondance remonte au temps où il est encore au Droit et rédige ses premières véritables critiques :

En sa lettre du 14 décembre 1922, il m'envoie un article sur Âmes et paysages de Léo-Paul Desrosiers (...). Ce sera le début d'une correspondance de dix ans et d'une collaboration continue où le jeune romancier va m'imposer le rôle de mentor ou d'un directeur intellectuel. Il me soumettra ses manuscrits; je m'efforcerai de lui enseigner sa technique d'écrivain sinon de romancier ${ }^{32}$.

Leurs échanges sont francs et Groulx ne se montre jamais complaisant, comme en témoigne joliment ce passage : 
Je n'irai pas jusqu'à dire, comme quelques-uns, que votre talent s'affirme plus à son aise dans la critique que dans le roman. Je persiste à considérer votre Terre vivante comme l'une des créations romanesques les mieux construites et les plus charmantes de notre littérature. Mais peut-être en vos Essais, comme en vos romans, subissez-vous jusqu'à l'excès la discipline, je devrais dire le joug de votre raison. On voudrait plus de spontanéité, plus de coups d'aile plus de vigueur, plus souvent l'écrivain qui écrit avec toutes ses facultés, toute son âme ${ }^{31}$.

C'est d'ailleurs à la demande de Lionel Groulx qu'il devient l'un des fondateurs et le premier rédacteur en chef de la nouvelle revue L'Action nationale qui paraît en janvier 1933 et dans laquelle il publie quelques poèmes et articles de fond. Comme il faut donner une orientation à la revue, il consulte certains membres influents du clergé, soit l'évêque de Rimouski, $\mathrm{M}^{\text {gr }}$ Courchesne ${ }^{34}$ et l'archevêque de Québec, qu'il connaît depuis son séjour à Ottawa où le futur cardinal Villeneuve ${ }^{35}$ avait exercé son sacerdoce. Son rôle à L'Action nationale est ingrat car il reste discret. Il identifie des sujets, trouve des auteurs et les convainc de faire parvenir un texte. Bernard sait être persuasif : «Vous êtes probablement très occupé, mais il n'y a que les hommes occupés à qui l'on demande du travail et des sacrifices $^{36} . »$ Le lendemain du jour de l'An 1933, par exemple, il rédige plus d'une douzaine de lettres à des collaborateurs potentiels.

L'examen de son imposante correspondance révèle qu'entre 1929 et 1933, il entretient plusieurs contacts personnels avec les écrivains. À Simone Routier, en qui il reconnaît une poétesse de talent, il donne des conseils ${ }^{37}$, tout comme à Clément Marchand qui restera un bon ami. Jean Bruchési fait aussi partie de ses amis intimes; ils militent ensemble pour la valorisation des livres canadiens. Avec Jean-Charles Harvey, les discussions portent surtout sur la difficulté de la critique littéraire. La plus riche correspondance littéraire est sans contredit celle avec Alfred DesRochers. Ils s'envoient des poèmes de leur cru en cherchant dans les commentaires de l'autre une critique sans complaisance et se développe ainsi, au fil des ans, 
une solide amitié pour le moins insolite : Bernard, catholique convaincu et DesRochers, paillard et bon vivant!

Malgré les encouragements admiratifs d'un DesRochers et d'un Dantin qui trouvent que ses «vers planent très haut et sont parmi les meilleurs, sinon les meilleurs de toute notre littérature versifiée ${ }^{38}$ ", Bernard ne publiera jamais de recueil de poésie ${ }^{39}$. Pourtant, il remporte un troisième prix lors du Concours de la Société des poètes. DesRochers, membre du jury, l'en informe le 8 avril 1929: "vous avez décroché le troisième prix avec votre poème "Que $j$ ' aime le changeant visage" (c'est au moins le titre qu'on a lu en public), le seul à mon goût qui méritait une récompense, parce qu'il avait de la forme et de la pensée ${ }^{40}{ }{ }$.

Surplus de travail, tourments familiaux, responsabilités accrues, Le Courrier annonce, le 22 décembre 1933 que «son directeur, M. Harry Bernard, assez gravement malade depuis jeudi dernier, a été incapable depuis de s'occuper de tout travail de bureau ${ }^{41} . \gg$ Il souffrait d'une otite interne et a dû subir une opération de la mastoïde. Selon ses propres dires, il «s'est balancé entre la mort et la vie, pendant quelque chose comme six semaines ${ }^{42} . »$ Il ne reviendra au travail que le 21 mai 1934 et de façon progressive. Jamais plus Harry Bernard n' acceptera ce rythme de travail infernal et 1934 marque ainsi une rupture dans sa carrière.

\section{Homme d'affaires et homme de conviction politique}

Cette rupture prend plusieurs formes. Il y a l'abandon de L'Action nationale, en décembre 1934 qu'il justifie dans deux lettres adressées à l'administrateur de la revue, le jésuite JosephPapin Archambault ${ }^{43}$. Abandon aussi de l'écriture romanesque : le roman sera pendant longtemps délaissé et ses critiques littéraires seront presque absentes jusqu'en 1941. L'homme de lettres prend une pause, l'homme d'affaires émerge. Nommé en 1935 gérant de la Compagnie d'imprimerie et comptabilité Ltée de Saint-Hyacinthe, qui édite Le Courrier de St-Hyacinthe ${ }^{44}$, Bernard veille désormais à la santé financière du journal et de son imprimerie. 
Sur un plan plus personnel, les documents disponibles nous amènent à penser qu'il dispose de peu de liquidités. Ainsi en juillet 1933, il se départit, pour un peu plus de mille dollars de ses cinquante actions du géant du nickel Inco $^{45}$. L'année suivante, il rachète les terrains que son père possède à Sainte-Geneviève dans l'ouest de Montréal, mais «au prix de 1 \$ et d'autres valables considérations que le vendeur aux présentes reconnaît avoir reçues de l'acquéreur (...) avant ce jour ${ }^{46} . »$ Il les revendra au moment de l'agrandissement de l'aéroport de Dorval. L'homme n'est pas riche et ne le sera jamais.

Pour mieux défendre les intérêts de son journal, il participe à la fondation, en 1932, de l'Association des hebdomadaires de langue française. Au départ, l'organisme vivote, aux dires mêmes de Bernard ${ }^{47}$. Néanmoins, «[s]es membres sentaient un vague besoin de s'unir, de se serrer les coudes, la nécessité de pouvoir présenter un front uni, à un moment donné, dans la poursuite d'une idée, la défense de certains intérêts ${ }^{48}$.» Mais progressivement, les rencontres des gérants et des directeurs développent des rapports d'amitié et de collaboration dont Bernard tirera beaucoup profit.

Son implication au sein de cette Association s'accroîtra. Membre du conseil d'administration en 1936-1937, il se préoccupe essentiellement du dossier des contrats collectifs régissant les salaires des ouvriers d'imprimerie. En octobre 1937, il se rend à Québec avec deux autres membres pour défendre leurs intérêts ${ }^{49}$. En effet, le contrat collectif qui régissait les salaires dans les imprimeries à Montréal et dans un rayon de cent milles prenait fin. Et ce contrat, qui stipulait un salaire minimum de vingt-quatre dollars par semaine pour quarante-huit heures de travail faisait bien l'affaire de l'Association, mais non des imprimeurs de Montréal. Ces derniers voulaient améliorer les salaires tout en incluant les imprimeurs ruraux afin d'éviter une concurrence déloyale. Bernard consacrera cinq éditoriaux à ce sujet et finira par obtenir gain de cause en maintenant le statu quo pour les imprimeurs ruraux ${ }^{50}$.

Mais ce n'était que partie remise. Dès juin 1939, la Fédération nationale catholique des métiers de l'imprimerie, de concert avec les 
maîtres imprimeurs de Montréal, repart en guerre contre les hebdomadaires ruraux en laissant courir le bruit qu'ils paient mal leurs ouvriers ${ }^{51}$. En vue de préparer la réplique des hebdomadaires, Bernard s'informe auprès des membres sur les salaires versés aux employés de leur imprimerie. Parallèlement, il mène une campagne d'opposition dans son journal en signant plusieurs éditoriau ${ }^{52}$ contre l'imposition d'un salaire minimum prohibitif. Ses prises de position l'ont sans doute aidé à devenir président de l'Association au mois d'août $1939^{53}$.

Au cours de son mandat d'un an, Bernard reprend l'alliance que l'Association avait scellée avec les journaux anglophones en 1937. Après avoir assisté, le 19 janvier 1940, à une réunion avec le ministre du Travail à Québec ${ }^{54}$, il poursuit des pourparlers avec R.A. Giles, représentant de la Canadian Weekly Newspaper Association, Quebec Division. Leur position commune s'avérera décisive, selon l'aveu même de politiciens libéraux alors au pouvoir à Québec ${ }^{55}$ : refus catégorique d'être assujettis aux contrats collectifs en vigueur ${ }^{56}$. Leur mémoire du 15 mars, adressé au premier ministre et au ministre du travail, portera fruit ${ }^{57}$.

Non seulement Bernard se comporte-t-il en homme d'affaires, mais il a modifié considérablement les orientations politiques de son journal. Encore en 1934, il affirmait la nécessité de maintenir les journaux «libres, indépendants des politiciens, capables de nommer franchement les choses par leur nom, d'apprécier les faits à leur valeur, les hommes à leur stature vraie ${ }^{58}$.» Même si, depuis son arrivée, son journal s'oppose à son concurrent Le Clairon de Télesphore-Damien Bouchard, membre influent du gouvernement Taschereau, et qu'il avoue lui-même dans ses mémoires qu'il a rompu avec la tradition familiale libérale ${ }^{59}$, Bernard avait réussi jusqu'alors à conserver une certaine neutralité60 qui agaçait pourtant certains conservateurs ${ }^{61}$. Mais la tourmente politique du milieu des années 1930 finit par l'entraîner. C'est ainsi qu'en juin 1935, il rencontre un membre important de l'organisation de Maurice Duplessis, Jean Martineau, et qu'il accepte d'organiser une rencontre politique à Saint-Hyacinthe ${ }^{62}$. Il ne faut plus s'étonner que le 15 novembre, 
Bernard se prononce nettement en faveur de l'Action libérale nationale, le parti des jeunes réformistes qui ont quitté le Parti libéral et qui allait sous peu se fusionner au Parti conservateur de Maurice Duplessis pour former l'Union nationale ${ }^{63}$. Les attaques du Courrier contre Le Clairon et Bouchard deviennent systématiques. Sa chronique «En marge des événements» se transforme rapidement en une tribune bêtement partisane ${ }^{64}$. À compter du 28 août 1936 et de façon presque régulière, il signe, sous un pseudonyme qui le rendra célèbre par la suite, soit «L'Illettré», une chronique qui s'intitulera, après un certain temps, «Les Cornichonneries du Clairon». Les accusations de patronage, de mensonges, d'irrégularités y abondent. Cette chronique cesse finalement de paraître le $1^{\text {er }}$ juin 1937. Tout cela fait peut-être partie d'une stratégie afin de mieux commercialiser son journal, qui dès l'automne 1935, se transforme pour attirer davantage le lecteur ${ }^{65}$. Nous sommes néanmoins loin des textes sociaux, culturels et de politique étrangère auxquels Bernard avait habitué ses lecteurs.

En fait, son approche partisane est telle qu'il en vient même à appuyer l'infâme loi du cadenas. Dans un éditorial du 2 décembre 1938, Bernard, après avoir précisé que Duplessis «ne rêve ni de dictature, ni de fascisme ${ }^{66}$ », conclut en affirmant : «Quoi qu'en pensent et disent les adversaires du gouvernement d'Union nationale, la loi du cadenas paraît excellente.» En outre, ses textes sont repris dans le journal de l'Union nationale, Le Temps, et il participe longtemps à la rédaction de discours et devient un ami de Daniel Johnson qui était d'ailleurs conseiller juridique de l'Association des hebdomadaires.

Bernard restera toute sa vie un fidèle allié de l'Union nationale. Il exigera même de son ex-collègue du Droit, Edgar Boutet, qu'il vient d'engager à titre de courriériste parlementaire à Québec, que ses articles soient favorables à l'Union nationale «et surtout antilibéraux ${ }^{67}$.» Cela vaudra d'ailleurs à Bernard certaines faveurs qui prennent la forme de voyages à l'étranger offerts par le gouvernement du Québec ${ }^{68}$. Comme le mentionnait J.-Ernest Laforce : 
C'est dommage qu'à l'Union Nationale on n'ait pas songé à utiliser vos talents sur un théatre [sic] plus vaste. Un jour où il y aurait eu moyen d'acquérir LA PRESSE à d'assez bonnes conditions, et LA PATRIE avec, j'avais fortement conseillé à M. Duplessis, s'il se décidait à acheter ces journaux de vous en confier la haute direction.L'idée lui avait souri, mais il ne se décida pas à ces transactions ${ }^{69}$.

En dépit des nombreux services rendus à l'Union nationale, Bernard restera confiné à Saint-Hyacinthe là où, semble-t-il, on avait besoin de lui ${ }^{70}$. Tout en maintenant ses activités politiques, Bernard prend un nouveau virage après son mandat de président de l'Association des hebdomadaires. L'intellectuel reprend du service.

\section{L'Illettré et le docteur en littérature}

En 1942, il obtient une licence en lettres qui s'avère utile pour son admission à la Société Royale du Canada. Comme l'admission est conditionnelle à l'appui d'au moins trois membres, il pouvait déjà compter sur son réseau d'amis : Jean Bruchési, Léo-Paul Desrosiers, Lionel Groulx, Donatien Frémont, Séraphin Marion et Maurice Hébert. C'est ainsi qu'il fut élu en mai 1943 en même temps que son bon ami Claude Melançon ${ }^{71}$. Déjà Esdras Minville lui demande son appui pour être admis à son tour en octobre $1943^{72}$.

Ses études doctorales prendront plus de temps. Toujours convaincu de l'importance du régionalisme, c'est à ce sujet qu'il consacre sa thèse. Bénéficiant d'une bourse de la Fondation Rockefeller, il entreprend, à l'automne 1943, un long voyage aux États-Unis afin de rencontrer des professeurs de littérature de différentes universités. Le journaliste profite de son périple pour rédiger des notes de voyage qui paraissent régulièrement dans $L e$ Courrier et qui mettent l'accent sur la présence canadienne-française en terre américaine. C'est en avril 1947 qu'il termine la rédaction de sa thèse intitulée «Le roman régionaliste aux États-Unis (19131940)» et en mars 1948 qu'il reçoit son doctorat. La maison d'Éditions Fides la publiera l'année suivante ${ }^{73}$. 
Tout en étudiant et en travaillant à plein temps au Courrier, Bernard lance, en décembre 1941, une chronique littéraire qui sera publiée pendant plus de trente ans, chronique qu'il signe de son pseudonyme, L'Illettré. Grâce à l'Association des hebdomadaires, Bernard peut compter sur la collaboration de nombreux directeurs de journaux qui diffusent sa chronique. Les ententes conclues resteront toujours les mêmes : «Vous publiez ou vous ne publiez pas, mais vous me payez à la fin de chaque mois $^{74} \gg$ : un dollar par chronique hebdomadaire. Plus d'une quinzaine de journaux accepteront; il s'agit notamment du Bien public, du Droit, du Travailleur de Worcester, de La Liberté et Le Patriote de Winnipeg, du Carillon d'Hawkesbury, du Lingot d'Arvida, de La Revue de Granby, de La Tribune de Sherbrooke et de La Parole de Drummondville. En quelques années, ses textes seront publiés à cent mille exemplaires.

Voilà donc qu'il revient à la critique, après dix ans d'absence. Cependant, ses textes ont changé. L'Illettré ne manifeste plus le même sens critique : il se contente plutôt de faire connaître les oeuvres et les écrivains au public. Le critique devient quelque peu publiciste, manifestant ainsi son sens du marketing et poursuivant son but d'inciter à la lecture et d'encourager le goût de la culture chez ses compatriotes. En tant que directeur du Courrier, sans doute a-t-il pris davantage conscience de l'impact du journal et des revues sur la population. Quand il demande un poème à Alfred Desrochers, en 1932, pour le faire paraître dans le premier numéro de l'Action nationale, il lui souligne que cela lui fera «quelque publicité» ${ }^{75}$. Ailleurs, il remercie Albert Lévesque, son éditeur, du communiqué relativement aux prix David qu'il a remportés et de la publicité qui en résultera ${ }^{76}$. Ces faits montrent bien que Bernard n'est pas seulement un écrivain mais un homme d'affaires soucieux de mettre ses écrits et ceux des autres en valeur.

Les textes de l'Illettré abondent en ce sens. Voyons brièvement en quoi ils consistent en s'arrêtant sur ses textes de 1949. Cinquante et un articles non plus concentrés sur la littérature canadiennefrançaise comme ceux de l'année 1929 étudiée plus haut, mais 
s'intéressant autant aux volumes et auteurs américains, latinoaméricains, européens, canadiens. Tous les genres y passent: de l'histoire d'une sainte Thérèse d'Avila à un court voyage en Amérique latine, en passant par Buffon, Balzac, Jean-Paul Sartre, Jane Austen, Margaret Mitchell, Emily Brontë, etc.

À compter des années 1960, plusieurs journaux cessent de publier sa chronique de sorte qu'en 1968, seulement sept d'entre eux continuent à faire paraître ses analyses. Quatre ans plus tard, ils ne sont plus que quatre, incluant bien sûr Le Courrier où il ne travaille plus depuis juin 1970. Pour des raisons de santé, Bernard met fin à cette chronique en mai $1973^{77}$ après en avoir rédigé plus de mille six cents.

Boulimique de lecture, Bernard possède une bibliothèque hors du commun ${ }^{78}$ qu'il a constituée par des commandes de livres régulières adressées à des libraires parisiens, new-yorkais et autres. Comme il l'affirme lui-même: «[J]'ai dans mes livres des collections qu'on ne trouve nulle part ailleurs : une centaine d'ouvrages sur l'Autriche-Hongrie, presque autant sur la Turquie, en français et en anglais $^{79} . »$

\section{Un retour au roman}

Une fois sa thèse terminée, Bernard dispose de plus de temps et reprend ses projets d'écriture. Il se lance dans la rédaction d'un nouveau roman, Les jours sont longs, qu'il soumet au concours du Cercle du Livre de France en 1950. Le choix d'un gagnant s'avère difficile. Le prix du Cercle du livre de France ayant été octroyé à Bertrand Vac pour Louise Genest, après plusieurs scrutins, les membres du jury recommandèrent que l'on publie également les autres finalistes ${ }^{80}$. C'est ainsi que les Éditions décidèrent de décerner le prix à Vac mais d'ouvrir un autre concours où les lecteurs eurent à se prononcer. En fait, dès l'été 1950, Pierre Tisseyre, directeur du Cercle du livre de France, avait pressenti la difficulté de choisir un gagnant; c'est pourquoi il avait proposé à Bernard de partager le 
prix : «[S]'il était impossible de réconcilier les différents partisans d'ouvrages se tenant de très près, [pourquoi] ne pas diviser le Prix entre deux gagnants ${ }^{81}$ ?» Comme cette suggestion ne fut pas retenue par le comité, il fallut trouver une autre solution, d'où cette idée d'un prix des lecteurs ${ }^{82}$ que Bernard remporta en janvier $1951^{83}$.

Déjà un nouveau manuscrit est en chantier: Une autre année sera meilleure. Sous un nom d'emprunt, il l'inscrit au même concours. En septembre 1951, Tisseyre le prévient que la lutte sera serrée. «Je crois toutefois que vous avez un concurrent très sérieux avec Évadé de la nuit qui est l'oeuvre d'un jeune employé de RadioCanada ${ }^{84}$.» André Langevin remporte le prix et Bernard devra se contenter de faire publier son manuscrit sous forme de feuilleton l'année suivante ${ }^{85}$.

À l'été 1952, il soumet un nouveau manuscrit, Le remplaçant, encore une fois sous un nom d'emprunt ${ }^{86}$. Mais le verdict est négatif et il échoue dans sa tentative de le faire publier par Paul Michaud de l'Institut littéraire du Québec ${ }^{87}$. Qu'à cela ne tienne, en 1953, il présente Dans le bleu du matin ${ }^{88}$. Le même sort l'attend; Langevin l'emporte encore avec Poussière sur la ville.

Ces échecs répétés mettront un terme à sa carrière de romancier. Bernard en sort meurtri et désabusé. Incapable de se remettre en question, il blâmera plutôt la télévision qui, selon lui, provoque une érosion culturelle dont les conséquences se manifestent par une diminution du lectorat et, en bout de course, le refus de ses manuscrits:

Avec l'avènement de la télévision, la littérature n'existe à peu près plus. Les éditeurs n'osent plus accepter un roman, un recueil de nouvelles ou de poèmes, etc. Les gens ne lisent plus, parce qu'ils regardent les images de la TV et les éditeurs ne veulent plus miser sur un manuscrit. [...] Nous avons l'impression que la télévision plus encore que la radio, est en train de tuer la littérature, et en même temps la culture générale et le goût ${ }^{89}$. 


\section{Le naturaliste}

Pour être marquée de plusieurs ruptures, la carrière de Bernard n'en conserve pas moins une constante : sa curiosité pour la nature. Selon lui, la connaissance des noms des plantes, des arbres et des animaux permet à l'écrivain de mieux situer le décor de ses récits. «Quand on décrit un paysage [...] que le cadre de l'oeuvre s'y prête, je demande simplement qu'on n'écrive pas de bêtises [...]. Et je crois que, pour décrire la nature canadienne sans commettre de sottises, il vaut mieux connaître cette nature que de ne pas la connaître ${ }^{90}$.» L'importance accordée à la description de la nature canadienne dans ses romans s'insère parfaitement dans l'approche régionaliste qu'il a défendue toute sa vie.

Mais ce n'est pas par devoir que Bernard accumule au fil des ans des connaissances en sciences naturelles. Ses vacances annuelles sont toujours consacrées à des excursions de pêche et de longues randonnées en forêt avec cartes et boussole. C'est là qu'il se ressource et refait ses forces. Quand l'Association des auteurs du Canada $^{91},-$ dont il fait partie et à laquelle il siège comme directeur - l'invite à une réunion officielle en 1931, il répond à Robert Choquette : «J'avoue que je me sens bien plus à l'aise à courir les champs et les grèves, de grandes bottes aux pieds ${ }^{92} . »$ Quand, en 1940, il doit organiser la tenue du prochain congrès de l'Association des hebdomadaires, il choisit Notre-Dame-de-Pontmain, dans les Laurentides, où il sera possible de «lancer la ligne» entre les délibérations.

Peu à peu, Bernard devient en quelque sorte un expert que l'on consulte occasionnellement. Ainsi Ovila Fournier, un ami et professeur à la faculté des sciences de l'Université de Montréal, lui demande des références sur les rongeurs ${ }^{93}$, ce qui lui vaudra une réponse fort bien étayée ${ }^{94}$. En 1950, c'est au ministère de la Chasse et de la Pêche qu'il envoie ses observations sur les écureuils, observations qui étonnent par la qualité des remarques soumises.

Bernard signera deux oeuvres majeures dans ce domaine, soit l'ABC du petit naturaliste canadien ${ }^{95}$ (textes de vulgarisation 
scientifique pour les jeunes, qui parait en plusieurs fascicules en 1936; d'autres s'y ajouteront au cours des années 1940) et Portages et routes d'eau en Haute-Mauricie (1952) dans lequel il relate ses nombreuses excursions. En outre, il contribue sporadiquement à de nombreuses revues tels La vie au grand air, la Revue chasse et pêche, Forêt et conservation, les Cahiers de chasse, Le Petit almanach du chasseur et du pêcheur. À la fin des années 1960, il parvient même à faire publier des articles de même teneur dans une revue de médecine, Le Mauricien médical.

Comme en témoigne la correspondance, sa carrière de naturaliste amateur a l'effet d'un baume sur les plaies laissées par les échecs du romancier au cours des années 1950 et par les critiques essuyées tout au long de sa vie. Même la carrière de journaliste semble l'avoir laissé amer. À Clément Marchand, il explique pourquoi il a pris sa retraite, après quarante-sept ans au Courrier: «[P]arce que j'ai 72 ans révolus depuis mai; que je veux recouvrir un peu de liberté; qu'on n'a plus la santé qu'on avait, malgré les vantardises et les illusions entretenues; parce que, surtout, je me sentais lassé, lassé d'écrire chaque jour des choses qui tombaient dans le tonneau sans fond ${ }^{96} . »$ Sa définition de la pratique journalistique n'est d'ailleurs guère plus reluisante : «[L]'art d'exprimer une opinion sur des sujets que j'ignore, qui ne m'intéressent pas, et sur lesquels, en somme, je n'ai aucune opinion. Triturer le tout, sans qu'il y paraisse, pour que le lecteur y trouve, avec sa satisfaction, logique, vraisemblance et crédibilitée ${ }^{97} . »$ Pourtant, on avait déjà souligné ses mérites de journaliste en lui décernant, en 1961, le prix Olivar Asselin ${ }^{98}$. Ce prix, dont il fut le septième récipiendaire et le premier à l'extérieur de Montréal, lui fut décerné par la Société Saint-Jean-Baptiste pour la qualité de la rédaction des articles et de la présentation typographique du Courrier de Saint-Hyacinthe.

Dès sa retraite, de sévères ennuis de santé compromettent les projets de voyage qu'il espérait faire avec sa deuxième femme, Alice Sicotte ${ }^{99}$. Au moment de partir pour Paris, un premier malaise cardiaque : «Je suis donc confiné dans ma petite maison de 
retraite paisible, avec instruction de bouger peu, de travailler le moins possible, de m'ennuyer le plus possible ${ }^{100}$.» Une thrombose coronarienne, en juillet 1972, laissera davantage de séquelles. Finalement, il s'éteint le 17 mai 1979.

La carrière de Bernard ne peut se résumer à une seule dimension. Certes, il fut journaliste catholique, défenseur d'une littérature authentiquement canadienne-française et naturaliste amateur toute sa vie, mais il fut aussi conservateur et unioniste à compter de 1935, gestionnaire d'une petite entreprise d'imprimerie, essayiste à la fin des années 1920, critique d'oeuvres occidentales pendant plus de trente ans, romancier acharné pendant une quinzaine d'années et étudiant universitaire au cours des années 1940.

Dans tout cela, ses romans, qui pourtant l'ont fait connaître à son époque et lui ont permis de figurer dans quelques dictionnaires, n'occupent finalement qu'une place secondaire. Cette biographie a bien démontré l'éclectisme de cet homme à travers une seule constante : l'écriture de cet homme de lettres. Plus de deux mille éditoriaux de toutes sortes, au delà de mille six cent chroniques de l'Illettré, des comptes rendus, des romans, des critiques littéraires, des nouvelles, des textes de vulgarisation scientifique, des poèmes, des contes, des discours politiques, des milliers de lettres à des clients, à des connaissances, à des collègues, à des amis, à des parents.

Bien que le nationalisme ait dominé sa pensée, en tant qu'intellectuel, ses réflexions dépassaient largement cette seule thématique. Il s'intéressait aussi à la poésie comme mode d'expression de l'âme humaine et avait une passion pour les sciences naturelles, pour la littérature étrangère (particulièrement américaine) et pour le développement d'un public lecteur éclairé. 


\section{NOTES}

1 Voir notamment, Jean-Paul Lamy, «L'homme tombé, roman de Harry Bernard», dans Maurice Lemire (dir.), Dictionnaire des oeuvres littéraires du Québec, Tome II, 1900-1939, Montréal, Fides, 1987 [1980], p. 573; Jacques Cotnam, «Bernard, Harry», dans The Oxford Companion to Canadian Literature, Toronto, Oxford University Press, 1997 [1967], p. 96-97; Réginald Hamel, John Hare et Paul Wyczynski, Dictionnaire pratique des auteurs québécois, Montréal, Fides, 1976, p. 57-58 (que ces auteurs reprennent dans le Dictionnaire des auteurs de la langue française en Amérique du Nord, Montréal, Fides, 1989, p. 120-121).

${ }^{2}$ Ainsi Pierre de Grandpré, et al., dans leur Histoire de la littérature française du Québec, Tome II (1900-1945), Montréal, Beauchemin, 1968, 390 p., ne lui consacrent même pas de notice biographique; Roger Duhamel, dans le Manuel de la littérature canadienne-française, (4éd.), Montréal, Éditions du Renouveau Pédagogique, 1967, p. 146, avait fait mieux de même que Gérard Tougas dans son Histoire de la littérature canadienne-française, Paris, Presses Universitaires de France, 1960, p. 245.

${ }^{3}$ Gaston Désaulniers, Étude de l'espace dans l'oeuvre romanesque d'Harry Bernard, mémoire de maîtrise (Littérature), Université du Québec à Trois-Rivières, 1974, 168 p. Un autre mémoire peut être également mentionné, celui de Janine Boynard-Frot, Structure du roman à thèse dans Les jours sont longs d'Harry Bernard, mémoire de mâitrise (Littérature), Université de Sherbrooke, 1974, 142 p.

${ }^{4}$ Robert Lahaise, Une histoire du Québec par sa littérature, 1914 1939, Montréal, Guérin, 1998, p. 343.

${ }^{5}$ Les Jean-Charles Harvey et Victor Barbeau sont beaucoup plus à la mode (voir Yves Lavertu, Jean-Charles Harvey. Le combattant, Montréal, Boréal, 2000, 462 pages; Michèle Martin, Victor Barbeau. Pionnier de la culture journalistique, Sainte-Foy, Presses de l'Université Laval, 1997, 216 p.; Chantale Gingras, Victor Barbeau. Un réseau d'influences littéraires, Montréal, l'Hexagone, 2001, 212 p. ${ }^{6}$ Daniel Chartier, L'émergence des classiques, Montréal, Fides, 2000, p. 288.

${ }^{7}$ Cette dernière est menée conjointement avec nos deux collègues Georges Bélanger et Yves Lefier. 
${ }^{8}$ France Ouellet, Répertoire numérique du Fonds Harry-Bernard, Montréal, Bibliothèque nationale du Québec, 1999, 200 p.

${ }^{y}$ Nous tenons à remercier madame Marcelle Bernard Morisset de nous avoir autorisés à consulter la correspondance de son père. Notons que Bernard a conservé une copie d'une grande partie des lettres qu'il a envoyées, ce qui rend ce fonds exceptionnellement riche. 10 Harry Bernard, Le Courrier de Saint-Hyacinthe [dorénavant CdSH], 3 novembre 1930, p. 1.

"Harry Bernard à Rémi d'Anjou, 12 mars 1964, Montréal, Bibliothèque nationale du Québec, Fonds Harry-Bernard, [dorénavant BNQ], 298/043/001.

${ }^{12}$ Manuscrit de Harry Bernard, Souvenirs d'enfance et de journalisme, $5^{\mathrm{c}}$ version, chapitre 4, p. 6, BNQ, 298/009/006.

${ }^{13}$ Ibid., p. 7.

${ }^{14}$ L'entreprise s'appelle la J.H. Bernard Co. Inc., Horace Bernard à Harry Bernard, BNQ, 17 juin 1929, 298/048/013.

${ }^{15}$ Horace Bernard à Harry Bernard, 11 décembre 1930, BNQ, 298/ 048/013.

${ }^{16}$ Harry Bernard, «Comment j'en vins à écrire», L'enseignement secondaire au Canada, vol. XXII (avril 1943), p. 552.

${ }^{17}$ Dossier Harry Bernard, Archives du Séminaire de Saint-Hyacinthe, [dorénavant ASSH], notes manuscrites de l'abbé Pierre-Athanase Saint-Pierre [s.d.].

${ }^{18}$ Dans les biographies, on fait souvent état de cet emploi de courriériste parlementaire. En fait, il ne le fut que quelques mois, avant de devenir adjoint au rédacteur en chef. Il faut admettre que luimême avait autorisé dans les biographies cette mention qui ne rend pas justice au travail varié et important qu'il effectua au Droit pendant quatre ans.

1" Harry Bernard, «Le vote des femmes», Le Droit, 30 novembre 1921, p. 3.

${ }^{21}$ Ibid.

${ }^{21}$ Notes manuscrites de l'abbé Pierre-Athanase Saint-Pierre, [s.d.], ASSH.

${ }^{22}$ Harry Bernard, Souvenirs d'enfance, op. cit., chapitre 6, p. 3 et 4, BNQ, 298/009/006.

${ }^{23}$ Encore aujourd'hui, plusieurs auteurs assimilent les deux termes que Bernard s'évertue à dissocier. Alors qu'on classe le plus souvent ses romans comme faisant partie du roman de la terre, il affirme avec véhẻmence qu'il n'a écrit que des romans régionalistes. Nous publie- 
rons prochainement un article sur ce sujet.

${ }^{24}$ Il s'agit des romans suivants : L'Homme tombé (1924), La Terre vivante (1925), La Maison vide (1926), La Ferme des pins (1930), Juana, mon aimée (1931), Dolorès (1932). Le recueil de nouvelles s'intitule La Dame blanche (1927) et le recueil de critiques, Essais critiques (1929). Un autre recueil de nouvelles paraîtra en 1935 (Montcalm se fâche), mais il s'agit en fait d'une reprise de nouvelles déjà publiées dans La Dame blanche, l'ouvrage ne comptant qu'un seul texte inédit, la nouvelle éponyme. Pour un aperçu des auteurs qui l'ont le plus influencé, voir Adrienne Choquette, «Harry Bernard», dans Confidences d'écrivains canadiens-français, Notre-Dame des Laurentides, Les Presses laurentiennes, 1976 [1939], p. 19-26. Voir aussi Harry Bernard à Jean Paré, $1^{\text {cr }}$ mars 1962, BNQ, 298/047/ 002.

${ }^{25}$ Dans une lettre que lui adresse Jean-Charles Harvey, le 5 octobre 1932, celui-ci, membre du jury, mentionne : «Inutile de te dire que j'étais heureux de contribuer à te donner une récompense pour ton travail, surtout pour ton progrès. La discussion a porté entre toi et Desrosiers (Nord-Sud). Il manquait à ce dernier ce que tu possèdes : l'action.», BNQ, 298/046/007.

${ }^{26}$ Voir Micheline Tremblay, La présence du cinéma dans le roman canadien-français (1896-1970), Thèse de doctorat, Université PaulValéry (Montpellier III), $550 \mathrm{p}$. Consulter à ce sujet le chapitre 3.

${ }^{27}$ Notamment dans Le Devoir, L'Action française, L'Apôtre, Le Canada français, La Revue nationale, la Revue de l'Université d'Ottawa, L'Almanach littéraire de l'Est, Books Abroad - A Quarterly Publication Devoted to Comment on Foreign Books, etc.

${ }^{28}$ Harry Bernard, Essais critiques, Montréal, Librairie d'Action canadienne-française, 1929, $196 \mathrm{p}$.

${ }^{29}$ Jean-Charles Harvey à Harry Bernard, le 5 novembre 1929, BNQ 298/046/007. À propos des difficultés de faire de la critique, Bernard écrira à Jeanne Grisé : «Savez-vous que cette question de l'appréciation des oeuvres devient fort épineuse? Ou les critiques feront leur travail avec conscience jugeant les livres avec ce qu'ils croient être leur valeur, et ils perdent leurs amis, s'attirent des inimitiés; ou ils essaieront d'être aimables pour tous, et passeront alors pour des nigauds, ne seront plus écoutés, desserviront les lettres.» Voir lettre de Harry Bernard à Jeanne Grisé, le 12 mars 1930, BNQ, 298/046/006. 
${ }^{30}$ Harry Bernard à Jean-Charles Harvey, le 6 novembre 1929, BNQ 298/046/007.

${ }^{31}$ Cela avait d'ailleurs été évoqué dans l'ouvrage récent dirigé par Jacques Michon, Histoire de l'édition littéraire au Québec au XX ' siècle. La naissance de l'éditeur, 1900-1939, vol. 2, Saint-Laurent, Fides, 1999, p. 259-261. Quelques années auparavant, Pierre Hébert avait également présenté quelques lettres de Bernard à Groulx, elles aussi localisées dans le Fonds Lionel-Groulx. Voir Lionel Groulx et L'Appel de la race, Montréal, Fides, 1996, p. 74-75.

${ }^{32}$ Lionel Groulx, Mes Mémoires, tome 2 : 1920-1928, Montréal, Fides, 1971, tome II, p. 135; voir aussi Harry Bernard, «Comment j'en vins...», loc. cit., p. 553.

${ }^{33}$ Lionel Groulx à Harry Bernard, 30 décembre 1929, BNQ, 298/ 046/006.

${ }^{34}$ Harry Bernard à $\mathrm{M}^{\mathrm{gr}} \mathrm{G}$. Courchesne, 21 décembre 1932, BNQ, 298/042/017.

${ }^{35}$ Harry Bernard à $\mathrm{M}^{\mathrm{gr}} \mathrm{R}$. Villeneuve, 21 décembre 1932, BNQ, 298/042/017.

${ }^{36}$ Harry Bernard à Antonio Barbeau, 2 janvier 1933, BNQ, 298/042/ 017.

${ }^{37}$ Entre septembre et décembre 1929, les deux s'écrivent près d'une quarantaine de lettres dans lesquelles il est question notamment de son ouvrage de poésie, L'immortel adolescent et de la critique que Bernard a rédigée à ce sujet.

${ }^{38}$ Alfred DesRochers à Harry Bernard, 5 septembre 1930 (voir aussi ses lettres du 7 et du 19 septembre 1930, BNQ, 298/045/007).

${ }^{39}$ Peut-être que la réaction mitigée de Lucien Rainier, appelé à commenter un recueil de ses vers, a-t-elle contribué à décourager Bernard dans cette voie. Voir Lucien RAINIER à Harry Bernard, 18 juillet 1932, BNQ, 298/046/007.

41) Alfred DesRochers à Harry Bernard, 8 avril 1929, BNQ, 298/045/ 007.

${ }^{41}$ «M. Harry Bernard absent du bureau», CdSH, 22 décembre 1933, p. 1.

42 Harry Bernard à Lucien [?], 2 juillet 1934, BNQ, 298/046/017.

${ }^{43}$ Bernard justifie son départ de plusieurs façons. Dans une première lettre datée du 30 juin 1934, il évoque le manque d'appui du comité de rédaction, le fait de ne pas être rétribué, le refus de prendre en considération ses nombreuses suggestions, un système de comptabilité déficient et bien sûr, le fait que sa santé ne lui permet plus de 
surcharge de travail. Voir lettre de Harry Bernard à Joseph-Papin Archambault, BNQ, 298/042/018. Dans une autre lettre du 7 décembre, Bernard évoque aussi le fait que la revue a publié, pendant son absence, un article inacceptable contre l'enseignement des sciences naturelles alors que son nom apparaissait encore comme rédacteur. Voir Harry Bernard à J.-P. Archambault, 7 décembre 1934, BNQ, 298/042/018.

${ }^{44}$ Harry Bernard à L.-A. Bastien, 17 mai 1935, BNQ, 298/043/006 : «depuis près de trois mois [...] mes loisirs se font de plus en plus rares. En plus de la direction du journal, j'ai à voir à toute l'administration de nos ateliers typographiques, ce qui veut dire donner de l'emploi à 15 hommes».

${ }^{45}$ Copie de la transaction effectuée le 13 juillet 1933, BNQ, 298/048/ 023.

${ }^{46}$ Copie de l'acte de vente des terrains de J. Horace Bernard à Harry Bernard, 12 octobre 1934, BNQ, 298/048/002.

${ }^{47}$ Harry Bernard, «L'Association des Hebdomadaires», CdSH, 9 octobre 1936, p. 3.

${ }^{48}$ Ibid.

${ }^{49}$ Voir Harry Bernard, «Les folichonneries du Clairon», $C d S H, 30$ octobre 1936, p. 3.

${ }^{50}$ Il s'agit des éditoriaux du 2 juillet 1937, du 14 janvier 1938, du 4 mars 1938, du 11 mars 1938 et du 18 mars 1938.

${ }^{51}$ Harry Bernard, «Les Hebdos», CdSH, 9 juin 1939, p. 3.

${ }_{52}$ Voir les éditoriaux du 9 juin, du 16 juin, du 23 juin, du 7 juillet, du 14 juillet et du 11 août 1939 du $C d S H$.

${ }^{53}$ J.M. McGee à Harry Bernard, 23 août 1939, BNQ, 298/046/018. ${ }^{54}$ Harry Bernard, «Communiqué confidentiel aux membres de l'Association», janvier 1940, BNQ, 298/043/003.

${ }^{55}$ Harry Bernard à Raymond Douville, 17 mars 1940, BNQ, 298/ 043/003.

${ }^{56}$ Harry Bernard et R.A, Giles, «Résolution approuvée par l'Association ... et la Canadian....», 10 mars 1940, BNQ, 298/043/ 003.

${ }^{57}$ Cette victoire semble cependant être remise en question dès l'année suivante. Le nouveau président de l'Association l'invite, sans succès, à se joindre à lui pour rencontrer le gouvernement en juin 1941. Voir lettre d'Harry Bernard à Charles Robidoux, 25 juin 1941, BNQ, 298/043/003. Et les pressions d'Alfred Charpentier, président de la Confédération des Travailleurs Catholiques du Canada, pour 
faire taire l'opposition de Bernard en mars 1941 démontrent que le débat se poursuit l'année suivante. Voir lettre d'Alfred Charpentier à $M^{g r}$ P. Desranleau, 3 mars 1941, BNQ, 298/043/004. Finalement, le fait qu'il soit président du comité des Contrats collectifs de l'Association encore en septembre 1950 laisse croire que cette question est toujours débattue. Mais la correspondance conservée n'en fait plus état tandis que Bernard est passé à d'autres champs d'intérêt.

${ }^{58}$ Harry Bernard, «De la presse de parti», CdSH, 26 octobre 1934, p. 1.

${ }^{54}$ Harry Bernard, Souvenirs d'enfance..., chapitre 2, p. 1 et 2, BNQ, 298/009/006.

${ }^{61}$ L'abbé Saint-Pierre avait bien saisi cette rupture provoquée par son engagement politique lorsqu'il écrivait : «Depuis quelques années, il est au service payé d'un parti politique, (....) ce qui est peu propre à inspirer confiance d'autant moins que plusieurs années durant il avait été indépendant des partis...». Notes manuscrites de l'abbé PierreAthanase SAINT-PIERRE, [s.d.], ASSH.

${ }^{61}$ Comme Jean-M. Carette du journal Le Guide de Beauce qui, mécontent d'un éditorial, lui écrit en octobre 1934 : «Vous méritez que toute la presse conservatrice rurale proteste contre votre ignorance en ce qui la concerne.» Lettre de J.-M. Carette à Harry Bernard, 28 octobre 1934, BNQ, 298/044/006.

(22Jean Martineau à Harry Bernard, 6 septembre 1935, BNQ, 298/ 046/021.

${ }^{63}$ Harry Bernard, «Parti national», CdSH, p. 3.

${ }^{64}$ En dépit de ses prises de bec avec Bouchard, Bernard sera reconnaissant envers lui. Ainsi, en faisant le bilan de sa carrière de journaliste, il déclara : «Le plus beau souvenir que je conserve de mes cinquante années de journalisme, c'est le témoignage que m'a rendu publiquement quelque temps avant son décès M. T.-D. Bouchard», CdSH , «Cahier spécial 125e anniversaire», 27 septembre 1978, p. 80.

${ }^{65}$ En laissant, par exemple, la première page aux nouvelles, pour déplacer la page d'opinion et d'analyse en page trois.

${ }^{66}$ Harry Bernard, «La loi du cadenas», CdSH, 2 décembre 1938, p. 3.

${ }^{67}$ Harry Bernard à Edgar Boutet, 11 novembre 1966, BNQ, 298/043/ 011.

${ }^{68}$ Ainsi en 1957, il participe au Congrès de la presse catholique de Vienne grâce à l'appui de Maurice Duplessis (voir lettre de Harry 
Bernard à Maurice Duplessis, 19 août 1957, BNQ, 298/046/001). Il avait participé à un autre congrès du même organisme en 1950 à Rome, probablement avec les mêmes appuis. En 1956, une note manuscrite nous indique que Daniel Jonhson serait intervenu pour lui permettre de se rendre au Festival du Livre de Caracas (voir lettre d'A. Plouffe à Harry Bernard, 29 octobre 1956, BNQ, 298/047/013) tandis qu'en 1959, les billets d'avion pour son voyage à Fort Chimo auraient été à la charge du ministère des Richesses hydrauliques dirigé par Johnson (voir Harry Bernard, Souvenirs d'enfance..., chapitre 8, p. 17, 298/009/006).

69 J.-Ernest Laforce à Harry Bernard, 17 mars 1961, BNQ, 298/046/ 013.

70. J.-Ernest Laforce à Harry Bernard, 3 juillet 1965, BNQ, 298/046/ 013.

${ }^{71}$ Cette société le déléguera au premier Festival du livre tenu à Caracas au Venezuela en 1956 à condition qu'il trouve lui-même l'argent pour défrayer le voyage. Bernard en profitera pour visiter sa fille aînée, Louella, qui réside au Venezuela avec son mari depuis le début des années 1950.

${ }^{72}$ Esdras Minville à Harry Bernard, 8 octobre 1943, BNQ, 298/047/ 001.

${ }^{73}$ Harry Bernard, Le roman régionaliste aux États-Unis (19131940), Montréal, Fides, 1949, 389 p.

${ }^{74}$ Harry Bernard à Roger Lussier, 5 juin 1968, BNQ, 298/046/017. À compter de 1968 , Bernard révisera finalement ses prix à la hausse en les majorant à trois dollars.

${ }^{75}$ Harry Bernard à Alfred DesRochers, 22 décembre 1932, BNQ 298/042/017.

${ }^{76}$ Harry Bernard à Albert Lévesque, 23 octobre 1934, BNQ 298/046/ 014.

${ }^{77}$ Harry Bernard à Marie-Anna Roy, 20 décembre 1973, BNQ, 298/ 047/011, et Nicole Turcotte à Harry Bernard, 21 mars 1973, 298/046/ 002.

${ }^{78}$ Bibliothèque qui impressionne les visiteurs de passage tel Wilson $\mathrm{O}$. Clough, professeur à l'Université du Wyoming. Voir lettre de Wilson O. Clough à Harry Bernard, 3 septembre 1949, BNQ, 298/044/001 . ${ }^{79}$ Harry Bernard à Gilbert Forest, 8 mars 1975, BNQ, 298/046/003. ${ }^{80}$ Voir le procès-verbal de la «Deuxième assemblée annuelle du jury» du Prix du Cercle du Livre de France tenue le 13 septembre 1950, 
Université de Sherbrooke, Archives du GRELQ (Groupe de recherche sur l'édition littéraire au Québec).

${ }^{81}$ Pierre Tisseyre à Harry Bernard, 21 août 1950, BNQ, 298/048/003. ${ }^{82}$ Les autres candidats à ce prix étaient Yves Thériault (Le dompteur d'ours) et Charles Hamel (Solitude de la chair).

${ }^{83}$ Pierre Tisseyre à Harry Bernard, 25 janvier 1951, BNQ, 298/048/ 003.

${ }^{84}$ Pierre Tisseyre à Harry Bernard, 14 septembre 1951, BNQ, 298/ 048/003.

${ }^{85}$ Harry Bernard, «Une autre année sera meilleure», Photo-Journal, XV, 43 (7 février 1952); XVI, 10 (19 juin 1952).

${ }^{86}$ Jean Bousquet à Harry Bernard, 16 juin 1952, BNQ, 298/043/010.

${ }^{87}$ Harry Bernard à Paul Michaud, novembre 1952, BNQ, 298/046/ 010.

${ }^{8 x}$ Jean Bousquet à Harry Bernard, 23 septembre 1953, BNQ, 298/ 043/010.

89 Harry Bernard à Jean Urruty, 6 janvier 1957, BNQ, 298/048/004; voir aussi Harry Bernard à Jean-Marie Gauvreau, 10 février 1957, BNQ, 298/047/013 et Harry Bernard à L.-A. Richard, 14 novembre 1959, BNQ, 298/047/008. Bernard n'avait pas tort de constater une diminution du nombre de romans publiés. Si l'on en croit Réjean Beaudouin, leur nombre annuel serait passé de vingt-sept entre 1939 et 1945 à dix-neuf entre 1947 et 1959, puis aurait remonté à quarante entre 1962 et 1965. Voir Réjean Beaudouin, Le roman québécois, Montréal, Boréal, 1991, p. 15.

") Harry Bernard à Alfred DesRochers, 20 février 1933, BNQ, 298/ 042/018; voir aussi Harry Bernard, «Histoire naturelle et littérature», L'Action nationale, vol. 1 n' 1 (janvier 1933), p. 18-28.

${ }^{\text {" }}$ Bernard est membre de cette Association au cours des années 1930 et il siège même sur le bureau de direction de l'organisme.

${ }^{92}$ Harry Bernard à Robert Choquette, 16 avril 1931, BNQ, 298/043/ 012.

${ }^{93}$ Ovila Fournier à Harry Bernard, 29 août 1935, BNQ, 298/046/003. ${ }^{94}$ Harry Bernard à Ovila Fournier, 30 août 1935, BNQ, 298/046/003. "5 Ces fascicules, publiés à Montréal aux Éditions Albert Lévesque sont intitulés : Le petit pêcheur, Le petit chasseur, Le petit oiseleur, Le petit entomologiste, Le petit fermier.

${ }^{* 6}$ Harry Bernard à Clément Marchand, 5 octobre 1970, BNQ, 298046-020.

${ }^{97}$ Idem. 
${ }^{98}$ Voir lettre de la Société Saint-Jean-Baptiste de Montréal à Harry Bernard, 28 février 1961, BNQ, 298/048/001.

${ }^{99}$ Sa première épouse était morte le 8 juillet 1957 et Bernard se remaria le 19 septembre de la même année. Voir lettre de Harry Bernard au Bureau des passeports du Canada, 21 août 1957, BNQ, 298/043/012. ${ }^{100}$ Harry Bernard à Gaston Roger, 28 septembre 1970, BNQ, 298/ 047/009. 